\title{
Outcomes of chronic subdural hematoma with preexisting comorbidities causing disturbed consciousness
}

\author{
*Yasuaki Abe, MD, Keisuke Maruyama, MD, PhD, Shigeomi Yokoya, MD, PhD, Akio Noguchi, MD, PhD, \\ Eishi Sato, MD, PhD, Motoo Nagane, MD, PhD, and Yoshiaki Shiokawa, MD, PhD \\ Department of Neurosurgery, Kyorin University School of Medicine, Tokyo, Japan
}

OBJECTIVE Chronic subdural hematoma (CSDH) is widely treated by drainage through a bur hole opening. However, whether and how preexisting comorbidities causing disturbance of consciousness affect patient outcomes remains unclear.

METHODS The authors analyzed 188 consecutive patients with CSDH who were surgically treated at the Neurosurgery Institute of the Kyorin University School of Medicine between 2010 and 2012 and followed them for more than 90 days. The mean patient age was 77.0 years (range 33-101 years) and 56 were women. Patient outcomes including modified Rankin Scale (mRS) score, postoperative morbidity and mortality, and recurrence 90 days after initial surgery were analyzed according to preexisting comorbidities causing disturbance of consciousness. The comorbidities observed in 46 patients (24\%) included dementia (30 patients), history of ischemic stroke (10 patients), psychiatric disorders (3 patients), and others (3 patients).

RESULTS Background characteristics of patients with comorbidities showed older patient age $(p<0.001)$, lower preoperative Glasgow Coma Scale score $(p<0.001)$, and higher preoperative mRS score $(p<0.001)$. The mean mRS score 90 days after the neurosurgical procedure was 1.2 in all 188 patients, which was significantly higher in those with comorbidities $(p<0.001)$. By 1 -way ANOVA with repeated measures, interaction existed between the presence of comorbidities and $\mathrm{mRS}$ score, and improvement of mRS score was observed in smaller proportions of patients with comorbidities $(p=0.002)$. By multivariate logistic regression analysis, the presence of comorbidities, patient age, reoperation for recurrence, and preoperative mRS score were significantly related to poor outcomes, defined as mRS score of 3 or more at 90 days after surgery. Postoperative morbidity $(p<0.01)$ and mortality $(p<0.01)$ were significantly higher in those with comorbidities, whereas the rate of recurrence of $\mathrm{CSDH}$ was not significantly different.

CONCLUSIONS The preexistence of comorbidities causing disturbance of consciousness affected severity and outcomes 90 days after surgical treatment of $\mathrm{CSDH}$, and comorbidities were also correlated with aging.

https://thejns.org/doi/abs/10.3171/2016.3.JNS152957

KEY WORDS chronic subdural hematoma; comorbidity; consciousness disturbance; outcome; surgery; trauma

$\mathrm{C}$ HRONIC subdural hematoma (CSDH) is one of the most common intracranial disorders and can usually result in great improvement with the application of a relatively simple surgical procedure. Bur hole irrigation and drainage has been widely performed as the gold standard of treatment. ${ }^{2,9,10,13}$ Although its surgical morbidity and mortality are generally low, the following factors are known to be associated with increased risk of morbidity or mortality: older patient age, lower level of consciousness, duration of the hematoma, midline shift, postoperative residual hematoma thickness, and nature of the intervention. ${ }^{1,4,6,8,10,12,14,16}$ Based on our recent clinical experience, we encountered some patients with preexisting diseases who exhibited poor recovery and longstanding disturbance of consciousness in spite of prompt and appropriate surgical treatment (Fig. 1). However, whether and how preexisting comorbidities causing disturbance of consciousness - such as dementia, psychiatric disorders, or previous history of stroke-affect patient outcomes remains unclear. Therefore, as factors associated with patient outcomes, we retrospectively analyzed whether comorbidities that could cause disturbance of consciousness contribute to the severity and therapeutic outcomes of CSDH.

ABBREVIATIONS CSDH = chronic subdural hematoma; GCS = Glasgow Coma Scale; $\mathrm{mRS}=$ modified Rankin Scale.

SUBMITTED December 21, 2015. ACCEPTED March 3, 2016.

INCLUDE WHEN CITING Published online May 27, 2016; DOI: 10.3171/2016.3.JNS152957.

* Drs. Abe and Maruyama contributed equally to this work. 

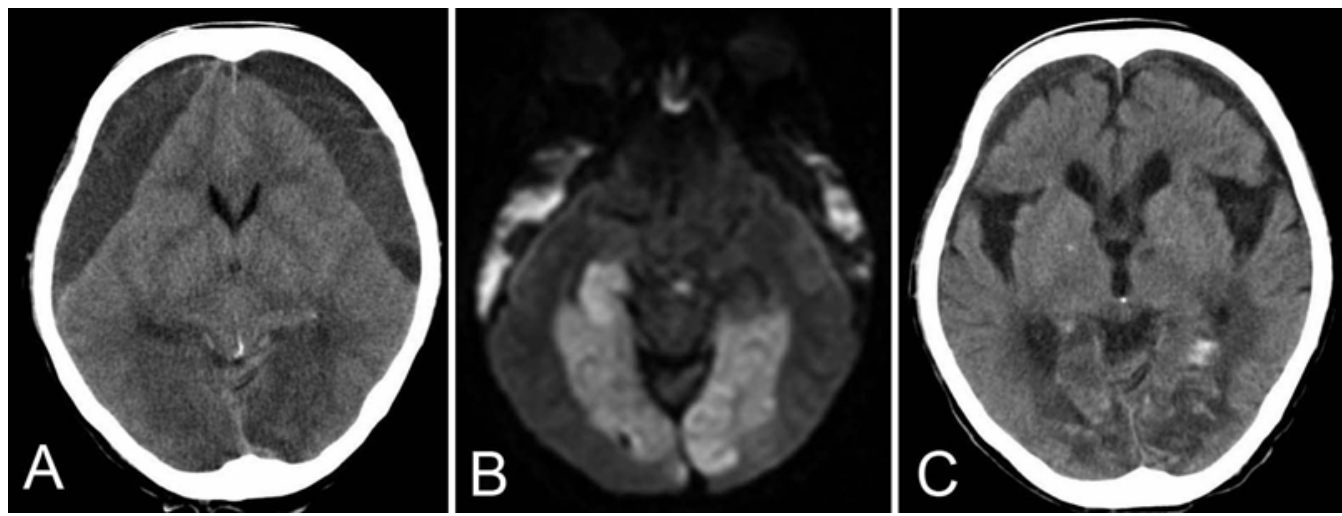

FIG. 1. Axial neuroimaging studies of an illustrative example of patients showing comorbidities causing disturbance of consciousness. A 77-year-old woman was transferred to our hospital because of disturbance of consciousness for 6 days. She had been hospitalized in another hospital for 8 years because of schizophrenia and a confused status. On arrival, she was in a comatose state with a GCS score of 3 . Her pupils were $5 \mathrm{~mm}$ and not reactive to light on either side. A CT image upon arrival (A) showed massive CSDH on both sides. Infarction in bilateral occipital lobes due to impending brain herniation was observed on diffusionweighted MRI (B). After bilateral subdural drainage with a bur hole opening performed on the day of presentation, she was still in an apallic state 90 days after surgery despite no recurrence of hematoma on a CT image (C).

\section{Methods}

\section{Patient Population}

We surgically treated 220 patients with CSDH at our institution between January 2010 and December 2012. Among them, follow-up for more than 90 days was available in 188 patients. These 188 patients were included in the analyses. The reason for loss to follow-up included transfer to another hospital (21 patients) and follow-up at another outpatient clinic (9), because of comorbidities in some patients. Characteristics of the patient population are shown in Table 1. The mean patient age was 77.0 years (range 33-101 years), and 56 patients (30\%) were women. Comorbidities causing disturbance of consciousness were defined as conditions that were known to be Glasgow Coma Scale

TABLE 1. Characteristics and outcomes of patients with CSDH according to comorbidities causing consciousness disturbance

\begin{tabular}{lccc}
\hline \multicolumn{1}{c}{ Variable } & $\begin{array}{c}\text { Group w/ } \\
\text { Comorbidity }\end{array}$ & $\begin{array}{c}\text { Group w/o } \\
\text { Comorbidity }\end{array}$ & p Value \\
\hline No. of patients & 46 & 142 & \\
\hline Mean patient age in yrs \pm SD & $83.6 \pm 10.1$ & $74.8 \pm 11.2$ & $<0.001$ \\
\hline Females (\%) & $17(37)$ & $39(27)$ & 0.25 \\
\hline Bilateral hematoma (\%) & $6(13)$ & $15(11)$ & 0.66 \\
\hline Mean preop GCS score \pm SD* & $12.6 \pm 2.0$ & $14.5 \pm 1.7$ & $<0.001$ \\
\hline Mean preop mRS score \pm SD & $3.1 \pm 1.0$ & $1.9 \pm 1.1$ & $<0.001$ \\
\hline Outcomes 90 days postop (\%) & & & \\
\hline Mean mRS score \pm SD & $2.6 \pm 1.5$ & $0.7 \pm 1.1$ & \\
\hline Poor outcome (mRS score $>2)$ & $24(52)$ & $12(8)$ & \\
\hline Postop complications & $7(15)$ & $2(1)$ & \\
\hline Postop deaths & $5(11)$ & $2(1)$ & \\
\hline Reop for recurrence & $5(11)$ & $171(12)$ & \\
\hline
\end{tabular}

* Preoperative GCS score was different from the GCS score used for the definition of the comorbidities, which was assessed before symptomatic presentation of $\mathrm{CSDH}$.
$(\mathrm{GCS})^{7}$ score of 14 or less by their families or other caregivers before symptomatic presentation of CSDH. Comorbidities were observed in 46 patients (24\%). In this subgroup of patients, the mean patient age was 83.6 years. Details of the comorbidities causing disturbance of consciousness included dementia (30 patients), history of ischemic stroke (10 patients), psychiatric disorders (3 patients), and others (3 patients; Table 2). Severity of dementia was classified according to the Clinical Dementia Rating. ${ }^{5}$ No patient had multiple comorbidities. In the other 142 patients (76\%) without comorbidities, the mean patient age was 74.8 years and 39 were female patients $(27 \%)$. As a surgical treatment, subdural drainage through a bur hole opening with or without irrigation was performed within 2 days of the diagnosis of symptomatic presentation of CSDH.

\section{Assessment of Outcomes}

The effect of comorbidities causing disturbance of consciousness on patient outcomes was retrospectively

TABLE 2. Details of the comorbidities causing disturbed consciousness in 46 patients

\begin{tabular}{cc}
\hline \multicolumn{1}{c}{ Comorbidity } & Value \\
\hline Dementia* $^{*}$ & 30 \\
\hline Alzheimer's disease $^{\prime}$ & 24 \\
\hline Vascular dementia & 4 \\
\hline Dementia w/ Lewy bodies & 4 \\
\hline Mean Clinical Dementia Rating \pm SD & $1.3 \pm 0.5$ \\
\hline History of ischemic stroke & 10 \\
\hline Mean mRS score \pm SD & $2.6 \pm 1.1$ \\
\hline Psychiatric disorders & 3 \\
\hline Schizophrenia & 1 \\
\hline Depression & 2 \\
\hline Others & 3 \\
\hline
\end{tabular}

* Two patients had mixed types of dementia. 
analyzed as were other factors, including age, sex, level of consciousness assessed with GCS, and modified Rankin Scale (mRS) score before surgery.11,15 Primary outcome was assessed by using the mRS score 90 days after the initial surgical intervention for CSDH. Outcome was defined as poor when the mRS score at 90 days was 3 or higher. ${ }^{8}$ Secondary outcome measures included postoperative complications, death, and repeated surgery for recurrence of CSDH within 30 days after the initial surgery. Relevant clinical information was retrospectively reviewed from medical records.

\section{Statistical Analyses}

For statistical analyses, the t-test was used for comparison of background variables. The chi-square test, 1-way ANOVA with repeated measures, and univariate and multivariate logistic regression analyses were performed to analyze factors related to patient outcomes. Only significant factors in univariate analysis were used for multivariate analysis. A p value $<0.05$ was used to indicate statistical significance. Statistical analyses were performed using SPSS (version 11, SPSS Institute).

The study protocol was approved by the institutional review board. Although a series of treatments had been performed after obtaining appropriate written informed consent from the patients, additional written consent for inclusion in this study was waived by the review board because of the retrospective and observational nature of the study.

\section{Results}

\section{Baseline Characteristics}

At the time of surgical treatment of 188 patients with CSDH, the mean GCS score was 12.6 (range 3-15), and the mean $\mathrm{mRS}$ score was 3.1 (range 1-5). None of the patients with comorbidities had a GCS score of 15 . The proportion of patients with comorbidities increased with age (Fig. 2 upper). In the group of patients with comorbidities, the ages ranged from 48 to 99 years (median 85 years). As a component of the comorbidities, dementia was observed only in patients 74 years of age or older, while psychiatric disorders and history of stroke were found in patients in their 40s and 50s. Patient age was significantly older, preoperative GCS score was significantly lower $(\mathrm{p}<0.001)$, and preoperative $\mathrm{mRS}$ score was significantly higher $(\mathrm{p}<$ $0.001)$ than in those without comorbidities (Table 1). The univariate logistic regression analysis indicated that the rate of preexisting comorbidities was significantly high in accordance with age $(\mathrm{p}<0.001)$. The number of patients with mRS scores greater than 2 before surgery was 37 $(80 \%)$ in the comorbidity group in contrast to $42(30 \%)$ in the noncomorbidity group. This difference was statistically significant $(\mathrm{p}<0.01)$. Other factors, including sex and bilateral presentation of CSDH, were not significantly different.

The proportion of patients with comorbidities among those who could not be followed for 90 days was significantly higher (15 [47\%] of 32 patients; $\mathrm{p}<0.01$, chi-square test) than those who could be followed (46 [24\%] of 188 patients).
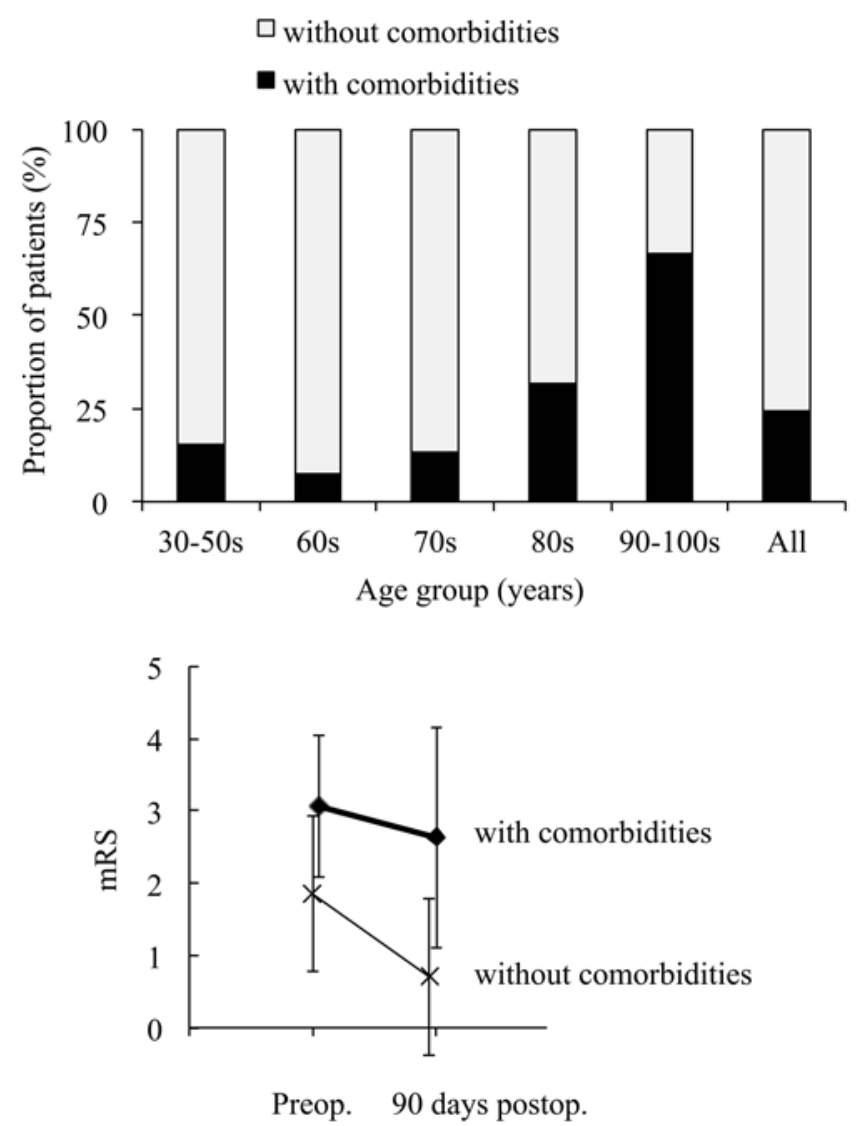

FIG. 2. Upper: Bar graph showing proportions of patients with preexisting comorbidities causing disturbance of consciousness according to age groups. For example, the range of " $60 \mathrm{~s}$ " was from 60 to 69 years of age. The proportions were higher in accordance with age, which was up to $67 \%$ in patients 90 to 101 years old, whereas it was only $7 \%$ in patients 60 to 69 years old. Lower: Graph showing the results of 1-way ANOVA with repeated measures that indicate change in the mRS score according to the presence of comorbidities. Improvement of mRS score was observed in significantly smaller proportions of patients with comorbidities $(p=0.002)$.

\section{Primary Outcome}

The mean mRS score 90 days after bur hole drainage was $1.2 \pm 1.5$ in all 188 patients. Primary outcome was poor in 36 patients (19\%), which included 24 (52\%) of 46 patients in the comorbidity group and $12(8 \%)$ of 144 patients in the noncomorbidity group. This difference was statistically significant $(\mathrm{p}<0.001)$. Worsening of $\mathrm{mRS}$ score was observed in 9 patients $(20 \%)$ in the comorbidity group, in comparison with only 1 patient $(0.7 \%)$ in the noncomorbidity group. This difference was statistically significant $(\mathrm{p}<0001)$. By 1-way ANOVA with repeated measures, there existed an interaction between the presence of the comorbidities and mRS score $(\mathrm{p}=0.002$; Fig. 2 lower). This meant improvement in mRS score was observed in significantly smaller proportions of patients with comorbidities. By univariate logistic regression analysis, patient age $(\mathrm{p}<0.001)$, preoperative GCS score ( $p<0.001)$, preoperative $\mathrm{mRS}$ score $(\mathrm{p}<0.001)$, and the presence of comorbidities $(\mathrm{p}<0.001)$ were significantly related to poor outcome (Table 3). Among the comorbidi- 
TABLE 3. Results of univariate and multivariate logistic regression analyses of factors associated with poor outcomes of $\mathrm{CSDH}^{*}$

\begin{tabular}{lcc}
\hline \multicolumn{1}{c}{ Factor } & Univariate & Multivariate \\
\hline Patient age & $<0.001$ & 0.011 \\
\hline Sex & 0.27 & - \\
\hline Bilateral hematoma & 0.99 & - \\
\hline Preop GCS score & $<0.001$ & 0.29 \\
\hline Preop mRS score & $<0.001$ & 0.02 \\
\hline Presence of comorbidities & $<0.001$ & 0.03 \\
\hline Presence of dementia & $<0.001$ & 0.97 \\
\hline
\end{tabular}

- = not applicable.

* All data shown are $p$ values.

ties, dementia alone was also significantly related to poor outcome. Sex and bilateral presentation of hematoma were not significant. Among these 5 significant variables, 3 factors except preoperative GCS score and the presence of dementia were also significantly related to poor outcomes by multivariate analysis. According to the similar $\mathrm{p}$ values, patient age, preoperative $\mathrm{mRS}$ score, and the presence of comorbidities were similarly related to outcomes.

\section{Secondary Outcomes}

Postoperative complications were observed in 9 patients (5\%) and included cerebral infarction or transient ischemic attack in 3 patients, pneumonia in 3 , and postoperative hemorrhage, probable pulmonary embolism, worsening of concomitant esophageal carcinoma, and cardiogenic shock in 1 patient each. Two patients had 2 complications, which were both fatal. The above-described complications were fatal except for cerebral infarction, transient ischemic attack, and postoperative hemorrhage in 1 patient each. A complication directly related to surgery was observed in only 1 patient, which was postoperative hemorrhage on the day after surgery, and no surgery-related mortality occurred. Reoperation for ipsilateral recurrence and contralateral growth was performed in $22(12 \%)$ and 6 patients (3\%), respectively. The rate of reoperation for ipsilateral recurrence was $11 \%$ in those with comorbidities and $12 \%$ in those without, which was not significantly different $(p=0.84)$. The rates of postoperative morbidity $(p<0.001)$ and mortality $(p=0.003)$ were significantly higher in those with comorbidities, while they were not significantly different in those with dementia alone $(\mathrm{p}=$ 0.62 and 0.84 , respectively).

\section{Discussion}

We found that the presence of preexisting comorbidities causing disturbance of consciousness affected severity and outcomes 90 days after surgical treatment of CSDH. Comorbidities also correlated with older age. Recovery of $\mathrm{mRS}$ score among the group with comorbidities was worse than that in the group without comorbidities 3 months after surgical treatment. Although this study would include a somewhat disparate group of patients, our clinical experience forced us to develop a new concept of "cerebral comorbidity," to which more attention should be paid. Although some previous studies reported older age as a risk factor for morbidity, ${ }^{6,10,12}$ little is known about the exact reason. Mental symptoms, such as somnolence, confusion, and memory loss, were pointed out as a possible reason. ${ }^{3}$ Our findings may explain one of the major reasons for poor outcomes in the elderly. Regarding extracranial comorbidities, renal failure, cardiac failure, deep vein thrombosis, and pneumonia were related to morbidity or mortality after treatment of CSDH, according to previous studies. ${ }^{6,10}$

Severity and poor outcomes in patients with CSDH with comorbidities can be explained by the following reasons. Although most patients without comorbidities could see neurosurgeons by themselves when they experienced any troubles, many patients with comorbidities had to depend on their families or other caregivers when visiting the surgical institute. As a result of the delay in hospital visits, they were diagnosed in a relatively deteriorated status (Fig. 1). Although CSDH is a "chronic" condition, longstanding elevated intracranial pressure by accumulation of hematoma in these patients would cause irreversible brain damage. In addition to poor condition before surgery, more frequent postoperative complications, such as ischemic stroke or pneumonia in those with comorbidities, observed in our series, would be another reason for poor outcomes. Indeed, their $\mathrm{mRS}$ scores did not recover to a similar extent as those without comorbidities, even after prompt and appropriate drainage of CSDH (Fig. 2).

Our findings appeared to conflict with findings from the previous study that indicated negative relationships between comorbidities and hospital mortality. ${ }^{12}$ However, this prior study had several things that were different from the current study. First, the proportion of "chronic" subdural hematoma in "any phase" of subdural hematoma was less than half. Second, eligibility for the prior study was 65 years or older. Thus, the patient population studied was considerably different, because our study included patients with only "chronic" subdural hematoma without an age restriction. Finally, various kinds of extracranial comorbidities of the heart, lung, liver, and so on were included as a "comorbidity score," and psychiatric disorders were not included in the prior study. All these differences might lead to the contradictory results.

This study has several limitations. First, assessment of $\mathrm{mRS}$ score before symptomatic presentation of CSDH was not necessarily performed by medical or co-medical staff. However, retrospective evaluation of the information from families or other caregivers of whether mRS had been a full score or not was relatively straightforward. Therefore, we considered that this method of assessment might not affect the results. Second, is the timing of outcome assessment at 90 days after surgery reasonable or not? Because most patients without complications could be discharged within 1 week after rapid recovery of their symptoms, we considered that assessment at 90 days might be appropriate to detect a delay in recovery. Another option would be the outcome assessment at 6 months, but in practice, we had completed postoperative follow-up within 6 months in 54\% of our patients because of the cure of subdural hematoma in most cases. Because a significantly higher proportion of our patients with comorbidities was lost to 90-day follow-up, mainly because of transfer to an- 
other hospital as reported, our analyses would be sufficiently modest to draw our conclusions.

\section{Conclusions}

As a result of evaluating outcomes of CSDH in relation to preexisting comorbidities causing disturbance of consciousness, most patients with comorbidities were in a severe state. The presence of comorbidities, correlated with aging, affected severity and outcomes 90 days after surgical treatment of CSDH. Particular attention should be paid to those with preexisting comorbidities causing disturbance of consciousness because they could lead to poor outcomes probably due to the delay in the diagnosis of CSDH.

\section{References}

1. Borger V, Vatter H, Oszvald Á, Marquardt G, Seifert V, Güresir E: Chronic subdural haematoma in elderly patients: a retrospective analysis of 322 patients between the ages of 65-94 years. Acta Neurochir (Wien) 154:1549-1554, 2012

2. Cenic A, Bhandari M, Reddy K: Management of chronic subdural hematoma: a national survey and literature review. Can J Neurol Sci 32:501-506, 2005

3. Fogelholm R, Heiskanen O, Waltimo O: Chronic subdural hematoma in adults. Influence of patient's age on symptoms, signs, and thickness of hematoma. J Neurosurg 42:43-46, 1975

4. Honda Y, Sorimachi T, Momose H, Takizawa K, Inokuchi S, Matsumae M: Chronic subdural haematoma associated with disturbance of consciousness: significance of acute-onchronic subdural haematoma. Neurol Res 37:985-992, 2015

5. Hughes CP, Berg L, Danziger WL, Coben LA, Martin RL: A new clinical scale for the staging of dementia. Br J Psychiatry 140:566-572, 1982

6. Javadi A, Amirjamshidi A, Aran S, Hosseini SH: A randomized controlled trial comparing the outcome of burr-hole irrigation with and without drainage in the treatment of chronic subdural hematoma: a preliminary report. World Neurosurg 75:731-736, 620-623, 2011

7. Jennett B, Teasdale G, Galbraith S, Pickard J, Grant H, Braakman R, et al: Severe head injuries in three countries. J Neurol Neurosurg Psychiatry 40:291-298, 1977

8. Leroy HA, Aboukaïs R, Reyns N, Bourgeois P, Labreuche J, Duhamel A, et al: Predictors of functional outcomes and recurrence of chronic subdural hematomas. J Clin Neurosci 22:1895-1900, 2015

9. Lind CR, Lind CJ, Mee EW: Reduction in the number of repeated operations for the treatment of subacute and chronic subdural hematomas by placement of subdural drains. $\mathbf{J}$ Neurosurg 99:44-46, 2003
10. Ramachandran R, Hegde T: Chronic subdural hematomascauses of morbidity and mortality. Surg Neurol 67:367-373, 2007

11. Rankin J: Cerebral vascular accidents in patients over the age of 60. II. Prognosis. Scott Med J 2:200-215, 1957

12. Rozzelle CJ, Wofford JL, Branch CL: Predictors of hospital mortality in older patients with subdural hematoma. J Am Geriatr Soc 43:240-244, 1995

13. Santarius T, Lawton R, Kirkpatrick PJ, Hutchinson PJ: The management of primary chronic subdural haematoma: a questionnaire survey of practice in the United Kingdom and the Republic of Ireland. Br J Neurosurg 22:529-534, 2008

14. Sucu HK, Gelal F, Gökmen M, Ozer FD, Tektaş S: Can midline brain shift be used as a prognostic factor to predict postoperative restoration of consciousness in patients with chronic subdural hematoma? Surg Neurol 66:178-182, 2006

15. van Swieten JC, Koudstaal PJ, Visser MC, Schouten HJ, van Gijn J: Interobserver agreement for the assessment of handicap in stroke patients. Stroke 19:604-607, 1988

16. Weigel R, Schmiedek P, Krauss JK: Outcome of contemporary surgery for chronic subdural haematoma: evidence based review. J Neurol Neurosurg Psychiatry 74:937-943, 2003

\section{Disclosures}

The authors report no conflict of interest concerning the materials or methods used in this study or the findings specified in this paper.

\section{Author Contributions}

Conception and design: Abe. Acquisition of data: Maruyama, Abe, Yokoya, Noguchi. Analysis and interpretation of data: Maruyama, Abe. Drafting the article: Maruyama. Critically revising the article: Maruyama, Nagane. Reviewed submitted version of manuscript: all authors. Approved the final version of the manuscript on behalf of all authors: Maruyama. Statistical analysis: Maruyama, Abe, Nagane. Administrative/technical/material support: Maruyama, Nagane. Study supervision: Noguchi, Sato, Nagane, Shiokawa.

\section{Supplemental Information \\ Previous Presentations}

Portions of this work were presented in poster form at the 73rd Annual Meeting of the Japan Neurosurgical Society, Tokyo, Japan, October 11, 2014.

\section{Correspondence}

Keisuke Maruyama, Department of Neurosurgery, Kyorin University School of Medicine, 6-20-2 Shinkawa, Mitaka-City, Tokyo 181-8611, Japan. email: kskmaru-tky@umin.ac.jp. 